\title{
Automatización de los procesos de inscripción y divulgación de la JUDC
}

\author{
Br. Michael Huete Matus, Br. Gustavo Carrillo Blandón. \\ Facultad Regional Multidisciplinaria-Matagalpa. ${ }^{1}$
}

Recibido 24 abril 2013- Aprobado 28 octubre 2013.

\section{RESUMEN}

La UNAN-FAREM Matagalpa contempla en sus actividades académicas, la Jornada Universitaria de Desarrollo Científico (JUDC); cada año se requiere gestionar la información de los trabajos inscritos; actualmente la inscripción de las investigaciones se maneja por medios escritos, presentando contratiempos; la divulgación se realiza de manera tradicional, sin embargo esto crea información poco precisa y no siempre disponible. El hecho anterior justifica la necesidad de evaluar el proceso actual de inscripción y divulgación de investigaciones de la JUDC, con el fin de detectar las dificultades; y así brindar alternativas de solución accesibles a la problemática actual. Este trabajo investigativo tiene un enfoque cualitativo con algunos elementos cuantitativos, según su diseño es experimental, por su aplicabilidad esta investigación es aplicada; por su profundidad es descriptiva y de corte transversal porque el estudio abarca un periodo determinado de tiempo. Las variables de estudio serán: los procesos actuales de inscripción y divulgación de investigaciones de la JUDC, dificultades en los procesos de inscripción y divulgación y alternativas de solución a las dificultades encontradas en los procesos de inscripción y divulgación de investigaciones de la JUDC. Las técnicas de recopilación de datos serán guías de encuestas, entrevistas, revisión bibliográfica y el método de prueba - error. El universo de estudio será la población universitaria de la FAREM Matagalpa que participará en la XIV Jornada Universitaria de Desarrollo Científico - 2012, distribuida en estratos poblacionales tales como estudiantes, tutores y asesores. La técnica de muestreo adecuada en ésta investigación será la de muestreo aleatorio estratificado.

Palabras clave: divulgación, inscripción, automatización, JUDC.

\section{INTRODUCCIÓN}

En la actualidad, la mayoría de las organizaciones especialmente las de tipo educativo, han ido evolucionando en su forma de trabajo; mejorando en gran medida la calidad de tareas asignadas, la planificación de procesos y la forma de gestionar y divulgar información. En este contexto, las Universidades nicaragüenses como UCA, UCC, UAM, UNAN-Managua y sus Facultades como Estelí, Chontales y Carazo; se han adaptado a las exigencias de la sociedad actual, incorporando el uso de medios automatizados, a sus procesos académicos, administrativos y de investigación, tratando con ello de mejorar la calidad de sus servicios y la eficiencia de las actividades de sus diversas áreas y dependencias.

Sin embargo, en la UNAN FAREM Matagalpa, las herramientas existentes no han sido explotadas en su totalidad, ya que aún existen unidades del centro que requieren de soluciones informáticas para mejorar el rendimiento en sus labores. Entre las áreas de trabajo que necesita incorporar mejoras en sus procesos, se encuentra la Dirección de Investigación. Esta representa una función esencial de la universidad; lo que significa que en esta área se maneja información importante para la institución.

La dirección de Investigación contempla en sus actividades académicas, la Jornada Universitaria de Desarrollo Científico - JUDC, "evento que reúne en espacio y tiempo a los jóvenes investigadores de la universidad y da lugar a la presentación de los resultados de investigación de los estudiantes, siendo esta una oportunidad para desarrollar el intercambio científico- técnico entre estudiantes, docentes, la comunidad universitaria y la sociedad" (Dirección de Investigación / JUDC, 2011).

\footnotetext{
${ }^{1}$ Trabajo dirigido por la licenciada Guisselle Raquel Martínez Ramos y la maestra MSc. Natalia Golovina.
} 
Durante cada Jornada se requiere gestionar la información de todos los trabajos inscritos; actualmente la inscripción de las investigaciones se maneja por medios escritos, presentando bastante contratiempos; en cuanto a divulgación se ha hecho de manera tradicional (avisos, murales, volantes pegados en las paredes); sin embargo esto crea información poco precisa y no siempre disponible; además se desconoce públicamente, el contenido de trabajos ganadores y las calificaciones que cada grupo obtiene.

El hecho anterior justifica la necesidad de evaluar el proceso actual de inscripción y divulgación de investigaciones de la JUDC, con el fin de detectar las dificultades; y así brindar alternativas de solución accesibles a la problemática actual.

\section{PLANTEAMIENTO DE PROBLEMA}

La dirección de Investigación de la FAREM Matagalpa representa una función esencial de la Universidad; con el fin de generar mecanismos que promuevan y divulguen los conocimientos adquiridos, resultados de investigaciones y proyectos emprendidos de los estudiantes, bajo la dirección de sus tutores respectivos.

Durante cada año la dirección de investigación contempla en sus actividades académicas, la Jornada Universitaria de Desarrollo Científico (JUDC). Cada Jornada requiere durante su proceso gestionar la información de todos los trabajos inscritos; actualmente la inscripción de las investigaciones se maneja por medios escritos, presentando bastantes contratiempos; en cuanto a divulgación se ha hecho de manera tradicional; sin embargo esto crea información poco precisa y no siempre disponible.

Se requiere evaluar el proceso actual de inscripción y divulgación de investigaciones de la JUDC, con el fin de detectar las dificultades, y así brindar alternativas accesibles que mejoren el proceso actual; por lo que es de suma importancia conocer ¿Cómo evaluar los procesos de inscripción y divulgación de investigaciones en la Jornada Universitaria de Desarrollo Científico JUDC, para su automatización en FAREM - Matagalpa año 2012?

\section{DESARROLLO}

\section{Dificultades en los procesos de inscripción y divulgación de investigaciones}

Económicas: La dificultad económica surge cuando se usa eficaz los recursos limitados para satisfacer las aspiraciones ilimitadas de una sociedad. Tras verificar que las aspiraciones son ilimitadas e inventariar los recursos existentes, se estudia el problema económico para demostrar que es necesario elegir y que la economía solamente puede crecer si los recursos se utilizan e incrementan eficazmente (Sada, 2007).

Este tipo de dificultades económicas están definidas por: gastos en accesorios y material de oficina, salario de personal, e inversiones de hardware y software; existen formas de solucionar las dificultades económicas como préstamos, renta de locales, reducir gastos entre otros. En la actualidad estas dificultades son las que se presentan mayormente en las organizaciones estas se deben principalmente a la falta de presupuesto, mala administración de recursos y la estructura.

Técnicas: Podemos decir que existe una dificultad cuando hay un conjunto de hechos y circunstancias que dificultan la consecución de un fin. Este tipo de dificultad es cuando un objeto o proceso no satisface las necesidades para la cual fue inventado entonces, hay una necesidad de crearlo. También cuando los objetos dejan de ser útiles por la evolución y la aparición de nuevas necesidades (Guzmán, 2012). La dificultad técnica evalúa los materiales, herramientas y procedimientos de una empresa, esta dificultad depende en gran medida de la Infraestructura tecnológica y capacidad técnica con que cuenta la institución. En la actualidad, toda empresa, no queda exenta de presentar dificultades técnicas que desequilibran el flujo de trabajo, sin lugar a duda se pueden observar problemas en el hardware y software 
con el que trabajan, lentitud en los procesos, errores de procesamiento; así como la falta de Integración de todas las áreas de la empresa.

Organizativas: En una visión estructural de las organizaciones, la dificultad es un problema que reduce la eficiencia. En el conflicto interno no es un problema, necesariamente, sino un componente natural e inevitable del proceso de la competencia interna por recursos, poder o influencia (Femenia, 2007). Las dificultades organizativas dependen en gran medida del mal aprovechamiento del recurso humano los cuales a su vez están vinculados a recursos materiales y financieros; produciendo como consecuencia el aumento de carga laborales del personal, alto consumo de tiempo, velocidad de procesamiento lenta, búsqueda y actualización de la información tediosa, falta de seguridad en almacenamiento de registros.

En la mayoría de las instituciones se hace necesario cumplir con una buena organización. Para llevar una buena organización se debe ordenar en todo momento las tareas y tener preparado los recursos en el momento necesario; los casos más comunes presentados en este tipo de dificultades son las diferencias interpersonales entre miembros de la institución; además de la inexistencia de conocimientos, habilidades y experiencia por parte del personal y la falta de comunicación entre las jerarquías de la empresa.

\section{Inscripción y divulgación electrónica con sistemas de automatización}

Inscripción electrónica: Representa las palabras o ideas mediante letras u otros signos gráficos utilizando medios digitales (WordReference, 2012). Este proceso es menos usual por la falta de capacitación de las personas, pero es muy útil ya que ahorra tiempo y espacio dentro de la institución. Una de las principales ventajas de utilizar este método es que proporciona información clara y precisa, agiliza el proceso de inscripción, procesa la información en período de tiempo corto, permite obtener información completa sobre trabajos terminados. Sin embargo, el uso de medios tecnológicos se convierte en una desventaja para algunas personas ya que no tienen el suficiente conocimiento para realizar estas operaciones.

Divulgación electrónica: Este medio de transmisión es dinámico, en el cual se puede crea, expresar, diseñar, informar y comunicar. El mensaje de los medios electrónicos es efímero, es decir, afecta las emociones del espectador durante muy pocos segundos y después desaparecen, la transmisión compromete más sentidos que la lectura por que añade tanto sonido como movimiento (Well, 2007). En los medios electrónicos el usuario final tiene la capacidad de interactuar con el medio es por ello que es considerado como dinámico dependiendo del grado de profundidad de información que se divulgue sobre este medio así será el impacto que tendrá sobre el público. Es de vital importancia para cualquier institución saber divulgar su información, la utilización de medios electrónicos proporciona una gran ventaja, ya que estos son una excelente forma de dar a conocer perfectamente el giro principal de una empresa; sin embargo el impacto que tendrá va en dependencia en la interacción que tenga el usuario con el medio, por lo tanto se puede convertir en un conveniente.

Definición de Automatización: La automatización es un sistema donde se trasfieren tareas de producción, realizadas habitualmente por operadores humanos a un conjunto de elementos tecnológicos que tratan de aplicar sistemas mecánicos, electrónicos y de bases computacionales para operar y controlar la producción (González Palomeque \& Verdugo Segovia, 2011).

\section{Ventajas y desventajas de la Automatización}

Según García (2010), las principales ventajas de la automatización son:

- Mejorar la productividad de una empresa reduciendo los costos de la producción y la calidad de la misma. 
- Realización de las operaciones imposibles de controlar intelectual o manualmente, mejorando la disponibilidad de los productos, logrando proveer las cantidades necesarias en el momento preciso.

- Permiten a una empresa enfrentar con éxito el mercado de trabajo e incorporarse al proceso productivo del país.

En definitiva se tienen que implementar los sistemas automatizados para mejorar el desarrollo de cada empresa; haciendo énfasis en la competitividad del mercado, costos de producción y sistemas productivo.

Entre las principales desventajas de la Automatización, tenemos:

- Una de las grandes desventajas seria que al automatizar las tareas, no se necesite de tanto personal de trabajo y ocasione desempleos.

- No toda persona está capacitada para utilizar maquinas inteligentes.

- A pesar de existir en el país, un elevado número de industrias en todos los campos de la producción, la gran mayoría no está en capacidad de competir en los mercados Internacionales, tanto en cantidad como en calidad.

- La implementación de estas tecnologías representa altos costos (García, 2010).

Una gran problemática en sí, sería el desempleo, debido a que muchas personas no cuentan con los conocimientos necesarios que actualmente demandarían las empresas con la adquisición de los nuevos recursos tecnológicos. La misión de cada empresa es ser mejor cada día, esto implica que tiene que mejorar la calidad de sus procesos mediante la automatización de sus equipo; representando grandes inversiones aunque muchas veces esto no garantice que será la mejor empresa tanto nacional como internacional.

\section{BIBLIOGRAFÍA}

Dictionary, T. F. (2012). Inscripción. Recuperado el 07 de 05 de 2012, de http://es.thefreedictionary.com/inscripci\%C3\%B3n

Dirección de Investigación / JUDC. (31 de Agosto de 2011). La Jornada Universitaria de Desarrollo Científico. Recuperado el 11 de Mayo de 2012, de http://www.unan.edu.ni/index.php?option=com_content\&view=article\&id=88\&Itemid=121

Femenia, N. (2007). Conflicto Organizacional. Recuperado el 23 de Mayo de 2012, de www.intermediacion.com/conflictoorganizacional.htm

García, E. J. (2010). Automatización. Recuperado el 17 de 05 de 2012, de www.slideshare.net/guest8f6e58/la-automatizacin-un-beneficio-o-un-maleficio-3792387

González Palomeque, C., \& Verdugo Segovia, J. (2011). Universidad Politécnica Salesiana. Recuperado el 16 de Mayo de 2012, de http://dspace.ups.edu.ec/bitstream/123456789/954/7/Capitulo_2.pdf

Guzmán, E. (2012). Problema Tecnológico. Recuperado el 16 de Mayo de 2012, de http://lawebtecnologica.wordpress.com/\%C2\%BFque-es-un-problema-tecnologico

Jara, E., \& Téllez, D. (2008). Sistema de Gerencia para Industrial IPMIS.Lima-Peru.

Sada, L. (2007). Definición del problema económico. Recuperado el 16 de Mayo de 2012, de www.peoi.org/Courses/Coursessp/mac/mac2.html

Well, W. D. (2007). Publicidad, principios y prácticas. México: Pearson Educación, séptima edición 624 pp.

WordReference. (2012). Definición de Inscripción. Recuperado el 07 de Mayo de 2012, de www.wordreference.com/definicion/inscripci\%C3\%B3n 